\title{
I SISTEMI ELETTORALI NELLE REGIONI A STATUTO ORDINARIO:
}

UN'ANALISI COMPARATA

di Nicolas Alejandro Cunial e Raffaele Terreo

Quaderni dell'Osservatorio elettorale n. 75, giugno 2016, pp 85-116 
Nicolas Alejandro Cunial ha curato il paragrafo 2 ad eccezione del sottoparagrafo sulle soglie di sbarramento, curato da Raffaele Terreo che ha inoltre redatto i paragrafi 1 e 3. Le conclusioni sono frutto di una riflessione comune.

\begin{abstract}
The electoral systems in the Italian regions: a comparative analysis.
Goal of this paper is to answer the question: what are the regional electoral systems in Italy now? After sixteen years of "electoral federalism», the electoral systems used to elect the President and the Regional Council have suffered more than a simple change over the years. Seven indicators are used to compare and contrast each of the systems: the broad family of systems it belongs to (majoritarian; proportional; mixed); the electoral constituencies; the structure of the electoral formula(s); the "second best candidate» clause; the majority prize; the electoral thresholds; the modes of vote's expression. Even though the goals that electoral reforms have set twenty years ago are still valid today - a higher bipolarity degree in the competition; high stability of the regional government; lower party fragmentation - a real progress has not been made towards these matters. The overview that emerges is clear: regions are adopting electoral systems that are more similar with respect to some indicators, and completely different with respect to others. Therefore, the aim of this paper is to clarify how current electoral rules work in the Italian regions, and to highlight the trends of electoral reforms.
\end{abstract}




\section{I sistemi elettorali regionali: dall'impianto nazionale al «federalismo elettorale»}

Nonostante la lettera dell'VIII Disposizione transitoria della Costituzione ${ }^{1}$ le Regioni a statuto ordinario sono entrate nella storia istituzionale italiana soltanto nel 1970, in occasione delle prime elezioni dei Consigli regionali, precedute dall'entrata in vigore della prima legge elettorale regionale, la 1. 108/1968, valida per le «Regioni a statuto normale» ${ }^{2}$, ossia non «speciale». Un ritardo figlio certamente delle divergenze che in sede di Assemblea Costituente avevano caratterizzato le posizioni delle varie forze politiche in materia di assetto da conferire al nascente Stato repubblicano; in quell'occasione, infatti, si era pervenuti ad una scelta compromissoria, né del tutto accentratrice né tantomeno federalista, ossia quella dello Stato regionale [Barbera, Fusaro 2014], prevedendo la nascita di Regioni intese come enti dotati di autonomia - ma non di sovranità - comunque titolari di potestà legislativa, seppur concorrente. Il reiterato rinvio del disciplinamento della materia elettorale regionale determinò una situazione di stallo che iniziò a sbloccarsi soltanto con il consolidamento dei rapporti tra DC e PSI nei governi di centrosinistra; risale infatti al Governo Moro III (particolarmente sensibile all'invigorimento istituzionale delle Regioni) la succitata 1. 108/1968.

In questo paragrafo verranno dunque descritti i caratteri fondamentali di questa legge, affrontando in seguito il mutamento delle regole elettorali regionali negli anni Novanta e analizzandone i precipitati normativi: la 1. 43/1995 e la 1. cost. 1/1999.

La l. 108/1968 e i suoi effetti sul sistema politico regionale. - La 1. 108/1968 istituiva per le Regioni a statuto ordinario un sistema elettorale proporzionale: la ripartizione dei seggi avveniva su base provinciale col metodo del quoziente Hagenbach-Bishoff; i seggi residui venivano assegnati ad un collegio unico regionale e successivamente ripartiti col metodo del quoziente naturale e dei più alti resti. L'elettore aveva la possibilità di attribuire fino a tre preferenze e non erano previste soglie di sbarramento esplicite. È evidente che quest'ultimo dato apriva la porta dei Consigli regionali ad un numero piuttosto elevato di formazioni partitiche, riproducendo di fatto una situazione - mutatis mutandis - analoga a quella che connotava il sistema partitico nazionale; non a caso la legge elettorale in questione era per larghi tratti ispirata a quella allora vigente per la Camera [Musella 2011], che aveva istituito, anch'essa, un sistema proporzionale con la possibilità di esprimere tre o quattro voti di preferenza: una scelta, questa, dettata da una precipua volontà politico-istituzionale emersa già in seno alla Costituente, ossia riprodurre «un sistema improntato alla responsabilità politica diffusa e al principio del compromesso» [Musella 2011, p. 164], che però connaturò come fortemente instabile l'azione di governo. Anche a livello regionale, conseguenza deteriore di un sistema siffatto fu rendere complessa la governabilità regionale, conferendo all'amministrazione regionale, spesso in balìa delle rivendicazioni delle formazioni consiliari anche minori, caratteri di inefficienza piuttosto

1 «Le elezioni dei Consigli regionali e degli organi elettivi delle amministrazioni provinciali sono indette entro un anno dall'entrata in vigore della Costituzione».

$2 \quad$ Art. 1, 1. 17 febbraio 1968 n. 108. 
marcati. Sarebbe stata l'onda del cambiamento politico-istituzionale a cavallo degli anni Ottanta e Novanta ad evidenziare la necessità di risolvere questo stallo, come vedremo nel paragrafo successivo.

I mutamenti degli anni Novanta: la l. 43/1995 e la l. cost. 1/1999. - La crisi elettorale ed organizzativa della partitocrazia italiana, che riguardava anche il «modo di governare» [Cotta e Isernia 1996] e il consociativismo il cui aspetto deteriore fu portato alla luce da Tangentopoli giocò un ruolo decisivo nell'alimentare «una forte domanda di stabilità governativa, di alternanza politico-programmatica, e di semplificazione del quadro politico» [Tarli Barbieri 2007, p. 41]. Tutto ciò costituì di certo il motore propulsivo della stagione referendaria del 1993, cui seguì l'istituzione di un sistema elettorale misto per Senato e Camera con la 1. 276/1993 e la 1. 277/1993 (ma anche l'introduzione dell'elezione diretta di sindaci e presidenti di Provincia ne fu un precipitato [Fusaro 1997]).

In questo contesto sembrò opportuno adeguare anche la normativa elettorale regionale, cercando di affiancare ad un pluralismo politico comunque da salvaguardare anche la stabilità dell'esecutivo regionale [Fusaro 2008], assicurando al Presidente eletto - come vedremo - una maggioranza certa [D'Alimonte 2000]: fu infatti adottata, in vista delle regionali dello stesso anno, la 1. 43/1995 (primo firmatario fu l'on. Tatarella), che novellò la norma precedente. Veniva introdotto un sistema misto, per cui l' $80 \%$ dei seggi era attribuito con formula proporzionale a liste provinciali ${ }^{3}$, il $20 \%$ con formula maggioritaria al listino regionale bloccato più votato, cui si collegavano più liste provinciali; era previsto un premio di maggioranza da attribuire al listino regionale vincente, in misura variabile: integrale - quindi il $20 \%$ del totale dei seggi - se le liste provinciali collegate avessero ottenuto meno del $50 \%$ dei seggi dei seggi totali del Consiglio nella sola parte proporzionale; dimezzato - quindi il $10 \%$ dei seggi - se avessero ottenuto almeno la maggioranza assoluta dei seggi (un «correttivo negativo» [Musella 2011] dagli effetti paradossali [Chiaramonte 2007] che poteva costituire un vero e proprio disincentivo a coalizzarsi [Pacini 2007]). In ogni caso alla coalizione vincente era garantito il 55\% o il 60\% dei seggi grazie all'attribuzione di un «premio aggiuntivo», ossia il possibile ampliamento dei seggi consiliari fino all'ottenimento delle quote ora citate, in base ai voti conseguiti dal listino regionale (rispettivamente, inferiori o superiori al 40\%), il cui capolista era de facto il candidato alla Presidenza della Giunta regionale. Si poteva inoltre esprimere un voto di preferenza, e si cercò di arginare la frammentazione partitica consiliare con una soglia di sbarramento del $3 \%$ per la lista provinciale, che però sarebbe entrata ugualmente in Consiglio se il listino regionale cui era collegata avesse ottenuto almeno il 5\% dei voti validi, venendo di fatto indotta a unirsi in coalizione, favorendo con ciò una dinamica potenzialmente bipolare.

Proprio questa assenza di soglia per le liste coalizzate non eliminava del tutto la frammentazione; di fatto, una reale stabilizzazione degli esecutivi regionali sembrò rag-

3 Prima i «seggi-interi» a livello circoscrizionale con metodo del quoziente Hagenbach-Bishoff, poi $\mathrm{i}$ «seggi-resto» a livello regionale, assegnati ad un collegio unico regionale, ripartiti tra le liste col metodo del quoziente e dei più alti resti ed infine restituiti alle circoscrizioni. 
giunta solo quattro anni più tardi con la 1. cost. 1/1999 [Pacini 2007], soprattutto perché estendeva dai primi due anni all'intera legislatura il legame tra Presidente e Consiglio (principio dell'«aut simul stabunt aut simul cadent»): le forze politiche di maggioranza sarebbero state da allora più restie a far venir meno il proprio appoggio al Presidente; inoltre quest'ultimo era «eletto a suffragio universale e diretto» ${ }^{4}$ ed aveva potere di nomina e revoca dei membri della Giunta: si trattava della «formula del governo di legislatura con elezione diretta del leader dell'esecutivo» [Olivetti 2002, p. 123], che, strettamente connessa alla riforma della legge elettorale [Tarli Barbieri 2007], era «innestata sulla formula elettorale introdotta già dalla legge 43/1995 (che) aveva trasformato la competizione proporzionale fra liste di partito in una competizione di tipo maggioritario fra coalizioni in vista della conquista di una sicura maggioranza assoluta nell'assemblea rappresentativa» [Fusaro 2007, p. 18].

La legge regionale, e non lo statuto ${ }^{5}$ (cui invece spetta la definizione della forma di governo, eventualmente alternativa a quella transitoria [Fusaro 2007] stabilita dall'art. 5 della 1. cost. 1/1999, nonché la determinazione del numero di seggi consiliari, entrambe evidentemente legate a doppio filo alla normativa elettorale regionale), deve provvedere a disciplinare il proprio «sistema di elezione» ${ }^{6}$, ma fino a quel momento rimane vigente la 1. 43/1995, con degli accorgimenti decisivi: i capilista dei listini regionali divengono de jure candidati alla Presidenza; viene introdotta la c.d. «clausola del secondo arrivato», cui è riservato di diritto un seggio in Consiglio (l'ultimo spettante alle liste provinciali ad esso collegate ovvero quello assegnato col resto in sede di collegio unico regionale $)^{7}$.

Ecco come si aprì la porta a quindici potenziali sistemi elettorali regionali differenti (per non citare quelli delle Regioni a statuto speciale), da definire in virtù di una potestà legislativa concorrente che però ha potuto fare riferimento ad una legge-cornice statale soltanto dal 2004, quando entrò in vigore la 1. 165/2004, che, da un lato, stabiliva in via esclusiva i principi fondamentali in materia di sistema di elezione regionale, dall'altro - di fatto - poneva pochi principi generali sul sistema elettorale in senso stretto, seppur di rilevante importanza: su tutti, la «individuazione di un sistema elettorale che agevoli la formazione di stabili maggioranze nel Consiglio regionale e assicuri la rappresentanza delle minoranze $\iota^{9}$. Un punto, questo, di primaria importanza - come vedremo - per la generalità dei sistemi elettorali regionali, seppur nel contesto di un loro «federalismo» ${ }^{10}$, e che infatti troverà precipua traduzione normativa nella previsione del premio di maggioranza ovunque, e quasi ovunque del tipo majority assuring.

Art. 122, comma 5, Cost., come modificato dall'art. 2, 1. cost. 22 novembre 1999 n. 1.

Corte cost. 13 gennaio 2004 n. 2.

Art. 122, comma 1, Cost., come modificato dall'art. 2, 1. cost. 22 novembre 1999 n. 1.

Art. 5, 1. cost. 22 novembre 1999 n. 1.

Art. 1, 1.2 luglio 2004 n. 165.

Art. 4, comma 1, lett. a, 1. 2 luglio 2004 n. 165.

R. D’Alimonte, «Il “federalismo” dei sistemi elettorali», in Il Sole 24 Ore, 31 maggio 2015. 


\section{L'attuale ingegneria elettorale nelle regioni a statuto ordinario}

Ricostruito il lungo processo legislativo che ha portato alla nascita del «federalismo elettorale» in Italia, ci addentriamo ora all'interno dei sistemi elettorali vigenti nelle regioni a statuto ordinario per comprenderne il funzionamento. La tabella 1 riporta le ultime leggi in materia di elezioni regionali varate dai rispettivi consigli.

Si è posta l'attenzione su quegli indicatori che fanno propriamente parte di un sistema elettorale e che non attengono invece alla «legislazione di contorno» e che la letteratura comparatistica sul tema è solita adoperare ${ }^{11}$. I sette indicatori sono: tipo di sistema; clausola "secondo arrivato»; le circoscrizioni; premio di maggioranza; formula elettorale; soglie di sbarramento e modalità di espressione del voto (a quest'ultimo indicatore è riservato il terzo paragrafo del presente elaborato).

Tab. 1 - I riferimenti normativi delle leggi elettorali regionali.

\begin{tabular}{ll}
\hline Regioni & Ultimo riferimento normativo \\
\hline Abruzzo & L.R. $02-04-13 \mathrm{n}^{\circ} 9$ \\
Basilicata & L. $43 / 1995$ \\
Calabria & L. R. $12-09-14 \mathrm{n}^{\circ} 19$ \\
Campania & L.R. $6-02-15 \mathrm{n}^{\circ} 3$ \\
Emilia-Romagna & L. R. $23-07-14 \mathrm{n}^{\circ} 21$ \\
Lazio & L.R. $19-04-13 \mathrm{n}^{\circ} 2$ \\
Liguria & L. $43 / 1995$ \\
Lombardia & L.R. $31-10-12 \mathrm{n}^{\circ} 17$ \\
Marche & L.R. $20-02-15 \mathrm{n}^{\circ} 5$ \\
Molise & L. $43 / 1995$ \\
Piemonte & L. $43 / 1995$ \\
Puglia & L.R. $10-03-15 \mathrm{n}^{\circ} 7$ \\
Toscana & L.R. $16-12-14 \mathrm{n}^{\circ} 79$ \\
Umbria & L.R. $23-02-15 \mathrm{n}^{\circ} 4$ \\
\hline
\end{tabular}

11 Si veda, tra gli altri, Gallagher M. e Mitchell P. (a cura di) [2005], The politics of electoral systems, Oxford, Oxford University Press. 
Tipo di sistemi, clausola "secondo arrivato" e circoscrizioni. - Anzitutto una distinzione necessaria: esistono sistemi elettorali top-down e sistemi elettorali bottom-up. I sistemi top-down aggregano i voti ottenuti nelle circoscrizioni e li ripartiscono con una formula preposta individuando così le quote di seggi spettanti alle liste e in quali circoscrizioni. Nei sistemi bottom-up, invece, i voti vengono immediatamente convertiti in seggi a livello circoscrizionale, ma le quote di voto non utilizzate e i seggi non assegnati vengono fatti confluire in un listone unico di livello superiore rispetto ai collegi stessi (nei casi qui in esame, il listone avrà dunque un confine circoscrizionale corrispondente alla regione). Di questo modello fanno parte i sistemi elettorali di Basilicata, Liguria, Molise, Piemonte, Puglia, Lazio ed Emilia Romagna. Le prime quattro perché mantengono in toto la 1. $43 / 1995$, le altre perché, sebbene abbiano adottato sistemi propri, non hanno modificato l'assetto elettorale proprio della Tatarella. Le altre Regioni, invece, hanno adottato sistemi elettorali di tipo top-down. Un'eccezione è rappresentata dalla Puglia, avendo questa Regione adottato, come poi vedremo, un'unica circoscrizione riferita quindi all'intero territorio regionale.

Sul tipo dei sistemi elettorali, non vi è molto da dire: come si evince dalla tabella 2, tutte le Regioni utilizzano un sistema elettorale di tipo misto. Infatti sono presenti tanto meccanismi di tipo proporzionale (formule proporzionali, collegi plurinominali) che di tipo maggioritario (formule maggioritarie, premi di maggioranza, collegi uninominali). Inoltre, la maggioranza dei sistemi elettorali analizzati, rientra nella tipologia dei proporzionali a premio di maggioranza [Chiaramonte 2005]. Rispetto a dieci anni fa, però, alcune delle Regioni che adottano la 1. 43/1995 non fanno più parte di questi sistemi poiché, come vedremo nel prossimo paragrafo, non utilizzano più un vero e proprio premio di maggioranza.

Per clausola del "secondo arrivato" s'intende quel particolare meccanismo, all'interno di un sistema elettorale, per cui un seggio viene riservato al candidato Presidente che ha ottenuto una quota di voti immediatamente inferiore rispetto al candidato risultato vincitore. Questo seggio può essere assegnato in due modi: l'attribuzione di diritto, perciò vi è un seggio prestabilito qual è quello riservato al Presidente eletto; l'attribuzione dell'ultimo dei seggi spettanti alla lista o insieme di liste collegate al candidato Presidente giunto secondo. Il primo metodo è previsto in Abruzzo e Veneto, unici sistemi tra quelli analizzati a riservare due seggi per i candidati alla presidenza della Regione che abbiano ottenuto il maggior numero di voti. Il secondo metodo viene impiegato invece nelle restanti regioni che prevedono questa clausola, utilizzata inoltre laddove è in vigore la $1.43 / 1995$ che non la conteneva, per effetto della legge costituzionale $n^{\circ} 1$ del 1999 . Le Regioni che invece si discostano dalla maggioranza sono Calabria, Umbria e Toscana. Se il sistema calabrese non la contempla - la questione è dinanzi alla Corte Costituzionale -, quello toscano e quello umbro utilizzano invece una clausola "estesa", poiché prevede l'assegnazione di un seggio a tutti i candidati alla presidenza della Giunta regionale «se collegati ad almeno un gruppo di liste che abbia ottenuto seggi» ${ }^{12}$.

12 Art. 21, comma 2 della legge regionale toscana 51/2014. Nel caso umbro la dicitura equivalente risulta essere: «collegati a coalizioni di liste o liste non unite in coalizione che abbiano conseguito almeno un 
ТАВ. 2 - Tipo dei sistemi elettorali e clausola "secondo arrivato" nelle regioni italiane.

\begin{tabular}{|c|c|c|}
\hline Regioni & Tipo & Clausola secondo arrivato \\
\hline Abruzzo & Misto & Sì \\
\hline Basilicata & Misto & Sì \\
\hline Calabria & Misto & No \\
\hline Campania & Misto & Sì \\
\hline Emilia-Romagna & Misto & Sì \\
\hline Lazio & Misto & Sì \\
\hline Liguria & Misto & Sì \\
\hline Lombardia & Misto & Sì \\
\hline Marche & Misto & Sì \\
\hline Molise & Misto & Sì \\
\hline Piemonte & Misto & Sì \\
\hline Puglia & Misto & Sì \\
\hline Toscana & Misto & Estesa \\
\hline Umbria & Misto & Estesa \\
\hline Veneto & Misto & Sì \\
\hline
\end{tabular}

In riferimento alle circoscrizioni, nella storia legislativa regionale si sono da subito imposti i collegi provinciali in virtù della maggior semplicità di utilizzare confini amministrativi preesistenti piuttosto che disegnarne ex novo. Dall'istituzione delle Regioni fino all'avvento del «federalismo elettorale» nel 1999, cioè per 29 anni, il disegno delle circoscrizioni per le elezioni regionali ha subito pochissime modifiche, rappresentando uno degli indicatori più stabili nel tempo e ciò non può che essere positivo poiché la consuetudine sembra inibire le volontà di manipolare il disegno dei collegi.

Come è possibile evincere dalla tabella 3 , però, tre Regioni hanno modificato il disegno delle circoscrizioni tramandato in questi anni da leggi nazionali: Calabria, Toscana e Umbria. La prima ha adottato una differente conformazione delle circoscrizioni riducendole da cinque a tre: la circoscrizione nord corrisponde alla provincia di Cosenza, la

seggio» come risulta dall'art. 2 comma 9 della legge regionale umbra 2/2010. 
circoscrizione centro contiene le provincie di Catanzaro, Crotone e Vibo Valentia, mentre la circoscrizione sud coincide con i confini della città metropolitana (che ha sostituito la provincia) di Reggio Calabria. La decisione, che si rifà alle tre provincie storiche della Calabria (quella di Vibo Valentia e di Crotone sono infatti nate nel 1992), è stata presa per omogeneizzare la quantità di popolazione presente nelle circoscrizioni. Tale scelta risulta opportuna in quanto permette che il "costo di voti", ossia la quantità di voti necessari ad una vittoria in un determinato seggio, sia uguale o molto simile tra le circoscrizioni.

ТАВ. 3 - Caratteristiche delle circoscrizioni nelle regioni italiane.

\begin{tabular}{|c|c|c|}
\hline Regioni & Circoscrizioni & $N^{\circ}$ collegi \\
\hline Abruzzo & Provinciali & 4 \\
\hline Basilicata & Provinciali & 2 \\
\hline Calabria & 2 provinciali e 1 multiprovinciale & 3 \\
\hline Campania & Provinciali & 5 \\
\hline Emilia-Romagna & Provinciali & 9 \\
\hline Lazio & Provinciali & 5 \\
\hline Liguria & Provinciali & 4 \\
\hline Lombardia & Provinciali & 12 \\
\hline Marche & Provinciali & 5 \\
\hline Molise & Provinciali & 2 \\
\hline Piemonte & Provinciali & 8 \\
\hline Puglia & Provinciali & 6 \\
\hline Toscana & Provinciali (Firenze divisa in 4 circoscrizioni) & 13 \\
\hline Umbria & Regionale & 1 \\
\hline Veneto & Provinciali & 7 \\
\hline
\end{tabular}

La Toscana, invece, ha lasciato inalterate le circoscrizioni provinciali ad eccezione di quella fiorentina, segmentata in quattro collegi differenti, per la medesima motiva- 
zione sopra citata e per ragioni di spazio interno alla scheda elettorale: infatti la scheda sarebbe diventata troppo lunga a causa della presenza delle "preferenze agevolate" in una circoscrizione vasta come quella fiorentina prima del suo spacchettamento. L'Umbria ha abolito completamente le due circoscrizioni provinciali di Terni e Perugia adottando un unico collegio regionale.

I premi di maggioranza: quanti sono, come operano. - Riteniamo prioritario definire, anzitutto, cosa sia un premio di maggioranza. Questo, perché sia tale, deve possedere tre proprietà: due necessarie e una accessoria. Le caratteristiche imprescindibili sono la «decisività» e la «sussidiarietà», mentre la terza è la «condizionalità» [Chiaramonte 2011]. Il primo requisito vuole che quando un premio di maggioranza scatti, debba effettivamente produrre una maggioranza. Ciò «non significa però che i sistemi elettorali che lo impieghino siano tutti necessariamente sistemi elettorali "a maggioranza assicurata" (majority assuring, con espressione inglese ormai di largo uso)» [Chiaramonte 2011, p. 27]. Legata alla prima proprietà vi è infatti quella della condizionalità: i sistemi elettorali possono prevedere premi di maggioranza vincolati alla necessità di raggiungere una certa soglia di voti o seggi affinché il meccanismo agisca. Il sistema elettorale derivato dalla 1. 148/1953, nota altresì come legge De Gasperi, ne è un esempio: esso assegnava un premio di maggioranza pari al $65 \%$ dei seggi alla lista o coalizione che avesse ottenuto la maggioranza assoluta dei voti. Peraltro una soglia tanto elevata da non permettere, in quell'unico anno di applicazione di tale meccanismo premiale, di ottenere il premio, per il mancato conseguimento dei voti necessari.

L'altra caratteristica è quella della sussidiarietà: questa qualità è fondamentale per la definizione di un premio di maggioranza e indica che quest'ultimo, perché sia tale, deve produrre delle modifiche alla distribuzione dei seggi operata dal sistema elettorale stesso mediante le formule di ripartizione. Un sistema a premio di maggioranza deve quindi essere così pensato: le formule elettorali distribuiscono i seggi secondo il loro meccanismo; si analizza se siano presenti e quali siano le condizioni dell'attribuzione del premio di maggioranza; in caso il premio di maggioranza scatti si modifica l'attribuzione dei seggi secondo le disposizioni del premio stesso.

Il premio non ha solo il pregio di creare una maggioranza, ma anche il merito «di generare una spinta all'aggregazione basata [...] sull'interesse e sulla convenienza» [Sartori 2013, p. 19] prima che le elezioni avvengano. Come lo stesso Sartori afferma successivamente, però, il merito può tramutarsi in difetto quando, una volta ottenuto il premio, i partiti coalizzatisi entrano in un clima di guerra politica sabotando dall'interno una maggioranza ottenuta in virtù di questo meccanismo. Un evento tutt'altro che inedito, a testimoniare come il premio di maggioranza rappresenti «uno degli elementi più controversi della transizione delle regioni italiane» [Musella 2011, p. 163].

La tabella 4 ci pone di fronte a un dato importante: 11 dei 15 sistemi elettorali regionali prevedono il meccanismo del premio di maggioranza. Un dato, questo, che si pone in linea con «l'analisi comparata dei casi (che) ha indubbiamente indicato l'Italia come il paese nel quale i sistemi a premio di maggioranza sono una sorta di "specialità" dell'ingegneria elettorale» [Chiaramonte 2011, p. 37], ma altrettanto in controtendenza 
rispetto alle analisi sui sistemi elettorali regionali finora compiute: rispetto al 2005, infatti, quando tutte le Regioni utilizzavano questo meccanismo [Chiaramonte, Tarli Barbieri 2007], il quadro attuale appare più frammentato.

Tutti i premi di maggioranza qui analizzati si basano su percentuali di voto ottenute da un candidato Presidente, ma vengono assegnati alle liste a lui collegate. Una precisazione è però necessaria: il premio di maggioranza, basandosi su condizioni di consenso attribuite al candidato Presidente, potrebbe scattare laddove la coalizione ad esso collegata non abbia ottenuto la percentuale di voti più alta. Questo può accadere dove si applica il voto disgiunto e dove vige ancora il listino regionale della legge Tatarella. Il caso del Piemonte nel 2010 è emblematico: Roberto Cota (CDX) superò appena, in termini di voti, l'avversaria Mercedes Bresso (CSX) nonostante la coalizione che la sosteneva avesse raccolto un maggior numero di consensi [Chiaramonte 2011].

La tabella 4 espone una varietà finora sconosciuta in ambito di premi di maggioranza regionali. Vi è da dire che talune Regioni utilizzano ancora il sistema elettorale della legge 43/1995 in toto, mentre altre limitano l'utilizzo di tale sistema ad alcune caratteristiche dello stesso, tra cui appunto il premio di maggioranza già descritto nel precedente paragrafo.

Il quadro che ne deriva offre quindi ad uno sguardo preliminare una visione piuttosto frammentata anche all'interno di tipi di premio già noti per il loro temporalmente ampio utilizzo. Vediamoli dunque nel dettaglio.

Tatarella limitato.In Basilicata, Lazio, Piemonte, Molise e Liguria vige un sistema elettorale derivato dalla legge 43/1995 il cui modello premiante non detiene però la caratteristica della decisività, configurando così un sistema elettorale privo di un reale premio di maggioranza e, perciò, determinando un sistema non più majority assuring. Questo perché nell'eventualità che la lista o coalizione collegata al candidato Presidente non ottenga, nonostante l'attribuzione integrale dei seggi facenti parte del listino regionale - che, ricordiamo, ammonta al $20 \%$ dei seggi totali in palio - la maggioranza assoluta dei seggi, non è possibile l'ampliamento del numero degli scranni consiliari, predeterminati e fissati dai rispettivi statuti regionali. In queste Regioni è perciò possibile che si crei un governo di minoranza. La Liguria, inoltre, prevede nel suo statuto una composizione del Consiglio regionale formato «da non più di trenta Consiglieri oltre al Presidente della Giunta» ${ }^{13}$. Tale dicitura comporta una possibile variabilità al ribasso dei seggi consiliari, potendo essere al di sotto dei trenta.

13 Legge statutaria 3 maggio 2005, art. 15 comma 3. 


Regioni Premio di maggioranza Quote di premio di maggioranza $\quad$ Majority assuring

\begin{tabular}{|c|c|c|c|}
\hline Abruzzo & Sì & $60 \%$ & Sì \\
\hline Basilicata & No & Tatarella limitato & No \\
\hline Calabria & Sì & Tatarella invertito & Sì \\
\hline Campania & Sì & $60 \%$ & Sì \\
\hline Emilia-Romagna & Sì & Tatarella invertito & Sì \\
\hline Lazio & No & Tatarella limitato & No \\
\hline Liguria & Sì & Tatarella & Sì \\
\hline Lombardia & Sì & Tatarella invertito & Sì \\
\hline Marche & Sì & $\begin{array}{l}\text { 1. Voti } \geq 40 \%=60 \% \text { Seggi; } \\
\text { 2. } 37 \% \leq \text { Voti }<40 \%=56,6 \% \text {; } \\
\text { 3. } 34 \% \leq \text { Voti }<37 \%=53,3 \%\end{array}$ & No \\
\hline Molise & No & Tatarella limitato & No \\
\hline Piemonte & No & Tatarella limitato & No \\
\hline Puglia & Sì & $\begin{array}{l}\text { 1. Voti } \geq 40 \%=58 \% \text { Seggi; } \\
\text { 2. } 40 \%>\text { Voti } \geq 35 \%=56 \% \text { Seggi; } \\
\text { 3. Voti }<35 \%=54 \%\end{array}$ & Sì \\
\hline Toscana & Sì & $\begin{array}{l}\text { 1. Voti } \geq 45 \%=60 \% \text { Seggi; } \\
\text { 2. } 45 \%>\text { Voti } \geq 40 \%=57,5 \% \text { Seggi }\end{array}$ & Sì \\
\hline Umbria & Sì & $60 \%$ & Sì \\
\hline Veneto & Sì & $\begin{array}{l}\text { 1. Voti } \geq 50 \%=60 \% \text { Seggi; } \\
\text { 2. } 50 \%>\text { Voti } \geq 40 \%=57,5 \% \text { Seggi } \\
\text { 3. Voti }<40 \%=55 \% \text { Seggi }\end{array}$ & Sì \\
\hline
\end{tabular}


Tatarella invertito. In Calabria, Emilia-Romagna e Lombardia, invece, a causa della determinazione di un numero fisso di seggi consiliari, il sistema prevede che i seggi necessari alla creazione di una maggioranza, qualora si presenti l'evento riportato per Basilicata, Piemonte, Molise, Liguria e Lazio vengano sottratti alle liste di minoranza, fino all'assegnazione del 55\% o 60\% (in base alla quota dei voti ottenuti, se inferiore o superiore al $40 \%$ ) dei seggi alla lista o coalizione del Presidente eletto.

Abruzzo, Campania, Umbria. Il premio di maggioranza utilizzato in queste Regioni è il più semplice e più forte: alla lista o coalizione collegata al candidato Presidente che ottiene più voti vengono riconosciuti, in virtù del premio, il 60\% dei seggi. Semplice perché non prevede quote diversificate di premio, ma solo, appunto, il $60 \%$ dei seggi che è in effetti l'ammontare comparativamente più elevato di seggi assegnabili fra le Regioni che adottano un premio di maggioranza. Forte poiché risulta essere il premio di maggioranza potenzialmente più distorsivo in termini di seggi assegnati sulla base dei voti conseguiti. Tale affermazione la si può così dimostrare: supponiamo che in tutte le Regioni in cui vige un sistema a premio di maggioranza - evitiamo di considerare la Toscana per semplificare l'esempio - i candidati Presidenti dichiarati vincitori e le coalizioni a essi collegate ottengano il 38\% dei voti. Questa quota di consensi è sufficiente a consentire che il premio di maggioranza scatti ovunque. Così in Abruzzo, Campania e Umbria i vincitori otterrebbero la più alta quota di seggi possibile, appunto il $60 \%$, mentre nelle restanti Regioni l'ammontare di seggi conseguiti sulla base dei rispettivi premi sarebbe inferiore.

Toscana. In questa Regione, con il $40 \%$ dei voti ma meno del $45 \%$ viene concesso un premio del $57,5 \%$ dei seggi, mentre se si supera la soglia del $45 \%$ dei voti, il premio diventa del $60 \%$. A prima vista potrebbe sembrare un sistema elettorale non majority assuring ma, in realtà, se nessun candidato Presidente ottiene il $40 \%$ dei voti, scatta il meccanismo del ballottaggio tra i due candidati che abbiano riportato le cifre elettorali più elevate. Al secondo turno, alla coalizione collegata al candidato Presidente risultato vincitore, sono assegnati il 57,5\% dei seggi.

Puglia, Veneto. Queste Regioni hanno un meccanismo premiante a tre soglie. La Puglia prevede per chi raggiunga o eguagli la soglia del $40 \%$ dei voti, lo scaglione più alto, un premio del $58 \%$ dei seggi. In Veneto, invece, il $40 \%$ è la soglia più bassa, sotto la quale si ottiene un premio del 55\% dei seggi. Il secondo scaglione della Puglia prevede che con soglie di voto inferiori al $40 \%$ ma superiori o uguali al 35\% si ottenga un premio del $56 \%$ dei seggi, mentre al di sotto del $35 \%$ dei voti il premio si riduce al $54 \%$ dei seggi. In Veneto, al contrario, gli altri due scaglioni sono più elevati: eguagliando o superando la quota del $40 \%$ dei voti validi, ma restando al di sotto del $50 \%$, il premio aumenta diventando del $57,5 \%$ dei seggi. In caso si raggiungesse o superasse il $50 \%$ dei voti, il premio si amplierebbe al $60 \%$ dei seggi.

Marche. Questa Regione si distingue da tutte per la sua diversa condizionalità: è infatti l'unico sistema non majority assuring pur dotato di un premio di maggioranza poiché questo non viene attribuito alla lista o coalizione vincitrice indipendentemente dal risultato. Il premio prevede infatti una soglia minima del $34 \%$ dei voti validi a cui corrisponderebbe, ammesso che non si eguagli o superi il $37 \%$ dei consensi, il 53,3\% 
dei seggi. Questo sistema elettorale è l'unico quindi a non garantire la formazione di una maggioranza in tutti i casi, lasciando così alla concertazione partitica il compito di formare una giunta secondo accordi e vantaggi tra liste in caso una maggioranza non sia formata dall'esito delle urne (o dal premio di maggioranza). Il secondo scaglione prevede che se si ottiene un ammontare di voti tra il $34 \%$ e il $37 \%$, il premio in seggi spettante sia del $56,6 \%$. Oltre il $37 \%$ dei voti, il premio aumenta al $60 \%$.

Se è vero che «la mancata previsione di una soglia minima di voti può essere interpretata come un segno della vocazione maggioritaria del sistema elettorale» [Musella 2011, p. 163], differente è stata l'interpretazione della Corte Costituzionale. Con la sentenza ${ }^{\circ} 1$ del 2014 infatti, in riferimento al premio di maggioranza della legge Calderoli, il sistema utilizzato per l'elezione del Parlamento tra il 2005 e il 2013, che attribuiva 340 seggi (poco meno del 54\% degli scranni) a chi avesse ottenuto più voti, indipendentemente dalla quantità di questi, recita:

«Tali disposizioni violerebbero l'art. 3 Cost., congiuntamente agli artt. 1, secondo comma, e 67 Cost., in quanto, non subordinando l'attribuzione del premio di maggioranza al raggiungimento di una soglia minima di voti e, quindi, trasformando una maggioranza relativa di voti, potenzialmente anche molto modesta, in una maggioranza assoluta di seggi, determinerebbero irragionevolmente una oggettiva e grave alterazione della rappresentanza democratica.

Esse, inoltre, avrebbero stabilito un meccanismo di attribuzione del premio manifestamente irragionevole, il quale, da un lato, sarebbe in contrasto con l'esigenza di assicurare la governabilità, in quanto incentiverebbe il raggiungimento di accordi tra le liste al solo fine di accedere al premio, senza scongiurare il rischio che, anche immediatamente dopo le elezioni, la coalizione beneficiaria del premio possa sciogliersi, o uno o più partiti che ne facevano parte escano dalla stessa. Dall'altro, provocherebbe un'alterazione degli equilibri istituzionali, tenuto conto che la maggioranza beneficiaria del premio sarebbe in grado di eleggere gli organi di garanzia che restano in carica per un tempo più lungo della legislatura.

Tale modalità di attribuzione del premio di maggioranza stabilita dalle predette disposizioni comprometterebbe, inoltre, l'eguaglianza del voto e cioè la parità di condizione dei cittadini nel momento in cui il voto viene espresso, in violazione dell'art. 48, secondo comma, Cost. La distorsione che ne risulta non costituirebbe, infatti, un mero inconveniente di fatto, ma sarebbe il risultato di un meccanismo irrazionale normativamente programmato per determinare tale esito».

In realtà tra le motivazioni della Corte Costituzionale rientra, non in modo secon- 
dario, la questione relativa alle funzioni del Parlamento, tra le quali quella di eleggere organi di garanzia costituzionale. Non avendo, le Regioni, funzioni simili, cade una delle motivazioni della sentenza.

Non secondaria appare la necessità di distinguere i premi di maggioranza utilizzati nei sistemi elettorali regionali da quelli invece adottati da Camera e Senato, in rapporto con la forma di governo in cui essi operano. Ben conosciamo infatti il funzionamento del principio simul stabunt simul cadent su cui poggia il legame tra Presidente della Giunta e il Consiglio regionale, che assicura «complementarietà e integrazione tra legge elettorale e forma di governo regionale» ${ }^{14}$. Diventa questa la base degli elementi a cui la Corte Costituzionale si è ispirata nell'adottare un diverso metro di giudizio, tradottosi in un «bilanciamento tra principio di rappresentatività e esigenza di governabilità diverso da quello applicato alle leggi elettorali di Camera e Senato» ${ }^{15}$, all'interno della sentenza $n^{\circ} 193$ del 24 settembre 2015. In questa sede, in riferimento al premio di maggioranza e alla soglia di sbarramento della legge elettorale lombarda, la Corte ha dichiarato inammissibile la questione di legittimità costituzionale in riferimento al premio. Non ha ritenuto, infatti, alla luce dei risultati delle elezioni lombarde del 2013, rilevanti le questioni relative alla possibile distorsione della presenza del voto disgiunto - poiché utilizzato minimamente dagli elettori e perciò non presenta un potenziale d'influenza sull'esito delle elezioni - né alla distorsione del principio di rappresentanza in virtù dell'assenza di una soglia minima di voti da conseguire a garanzia della fruibilità del premio. Tale sentenza è importante perché costituisce un possibile precedente in direzione di una costituzionalità dei premi di maggioranza regionali. In realtà, però, è possibile pensare che tale questione sia temporalmente limitata: tra le motivazioni della sentenza in riferimento all'assenza di una soglia minima di voti, la Corte dichiara che tale questione non è rilevante poiché non si è prodotto, con le elezioni del 2013, una distorsione grave del principio di proporzionalità, avendo il candidato Presidente vincitore, e così la coalizione ad egli collegato, superato la soglia del $40 \%$, ottenendo per effetto del premio il $60 \%$ dei seggi. Il dubbio s'impone di conseguenza nella eventualità che, in futuro, possa accadere, come per le elezioni nazionali del 2013, che una lista o coalizione di liste ottenga un premio di maggioranza in virtù del conseguimento da parte del candidato Presidente di una porzione minimale di consensi.

Viene poi in soccorso alle motivazioni della sentenza 193/2015, la legge $n^{\circ} 165$ del 2 luglio del 2004, che all'articolo 4 - disposizioni di principio, in attuazione dell'articolo 122, primo comma, della Costituzione, in materia di sistema di elezione - recita:

«Le regioni disciplinano con legge il sistema di elezione del Presidente della Giunta regionale e dei consiglieri regionali nei limiti dei seguenti princìpi fondamentali:

a) individuazione di un sistema elettorale che agevoli la formazione di sta-

14 Cozzi A. O. [2015], «La Corte non si esprime sui premi di maggioranza regionali», in Forumcostituzionale.it, p. 2.

$15 \quad$ Ibidem. 
bili maggioranze nel Consiglio regionale e assicuri la rappresentanza delle minoranze;

b) contestualità dell'elezione del Presidente della Giunta regionale e del Consiglio regionale, se il Presidente è eletto a suffragio universale e diretto. Previsione, nel caso in cui la regione adotti l'ipotesi di elezione del Presidente della Giunta regionale secondo modalità diverse dal suffragio universale e diretto, di termini temporali tassativi, comunque non superiori a novanta giorni, per l'elezione del Presidente e per l'elezione o la nomina degli altri componenti della Giunta;

c) divieto di mandato imperativo».

La lettera a) del comma unico dell'art. 4 stabilisce dunque la necessità, specialmente in ambito proporzionale, dell'adozione di un premio di maggioranza che permetta la formazione di una maggioranza consiliare. Tale principio, difeso dalla sentenza 193/2015 già citata, non garantirebbe però a priori la costituzionalità stessa dei premi di maggioranza in ambito regionale poiché non è possibile stabilire generalmente, anche alla luce delle sentenze emesse dalla Corte Costituzionale ${ }^{16}$, la costituzionalità di tali meccanismi. Una possibile soluzione potrebbe essere rappresentata dal meccanismo del ballottaggio presente nella legge toscana: a tal proposito, un primo riscontro lo si avrà con il giudizio di costituzionalità della legge elettorale italiana 52/2015 il cui meccanismo di ballottaggio è identico a quello toscano. In definitiva, però, si dovrà attendere che la Corte si esprima per ogni legge elettorale regionale, avendo queste premi di maggioranza piuttosto differenti tra loro.

Per concludere, riteniamo importante ribadire che se è vero che il premio di maggioranza ha lo scopo di favorire la nascita di maggioranze, come abbiamo visto, e di plasmare il contesto politico in un assetto bipolare, questi obiettivi appaiono distanti da una loro piena realizzazione a causa di una sempre più alta frammentazione partitica, favorita da soglie di sbarramento inadeguate e un'amplissima componente proporzionale [Chiaramonte, Tarli Barbieri 2011].

Le formule elettorali. - Le formule di ripartizione dei seggi sono la base matematica di un sistema elettorale. Sono, di fatto, i metodi di conversione matematica dei voti espressi in seggi, ovvero ciò che discrimina, a monte, un sistema elettorale dall'essere di tipo proporzionale o maggioritario.

Appare evidente che nel caso dei sistemi misti non vi siano formule peculiari, ma una congiunzione tra due metodi di ripartizione facenti parte dei due tipi di sistemi elettorali sopra citati.

Quota proporzionale. Dalla tabella 5 risulta che vi siano Regioni che sfruttano due formule: sono quelle che utilizzano interamente o in parte la legge ${ }^{\circ} 43$ del 1995. Questo perché, al contrario dei sistemi top-down, le fasi procedurali di ripartizione dei seggi sono appunto due: una a livello di circoscrizione, la seconda a livello regionale 
dove confluiscono quote di voto inutilizzate e seggi non assegnati.

Nelle quote proporzionali dei sistemi elettorali presi qui in esame vi sono soltanto tre formule che si riscontrano: la formula D'Hondt e la Hagenbach-Bishoff, in qualche caso affiancata dalla formula Hare.

Quota maggioritaria. Come per la quota proporzionale, anche la quota maggioritaria richiede una precisazione: i seggi riferiti a questa quota sono solamente quelli del Presidente eletto e, laddove il sistema elettorale preveda un seggio di diritto, del candidato Presidente che ha ottenuto la quota immediatamente inferiore rispetto al Presidente eletto. Si tratta di considerare quindi la Regione come un'unica circoscrizione e determinare chi ha preso più voti. A questo punto, le strade si dividono in base alla formula maggioritaria adottata nel sistema elettorale.

Tab. 5 - Le formule di ripartizione dei seggi rispettivamente in quota proporzionale e maggioritaria per ciascun sistema elettorale regionale.

\begin{tabular}{|c|c|c|}
\hline Regioni & Formula di ripartizione in quota proporzionale & Formula di ripartizione in quota maggioritaria \\
\hline Abruzzo & D’Hondt & Plurarility \\
\hline Basilicata & Hagenbach-Bishoff; Hare & Plurarility \\
\hline Calabria & Hagenbach-Bishoff; Hare & Plurarility \\
\hline Campania & D'Hondt & Plurarility \\
\hline Emilia-Romagna & Hagenbach-Bishoff; Hare & Plurarility \\
\hline Lazio & Hagenbach-Bishoff; Hare & Plurarility \\
\hline Liguria & Hagenbach-Bishoff; Hare & Plurarility \\
\hline Lombardia & D’Hondt & Plurarility \\
\hline Marche & D'Hondt & Plurarility \\
\hline Molise & Hagenbach-Bishoff; Hare & Plurarility \\
\hline Piemonte & Hagenbach-Bishoff; Hare & Plurarility \\
\hline Puglia & Hagenbach-Bishoff & Plurarility \\
\hline Toscana & D’Hondt & Majority "corretto" \\
\hline Umbria & Hagenbach-Bishoff & Plurarility \\
\hline Veneto & D'Hondt & Plurarility \\
\hline
\end{tabular}


La tabella 5 ci pone di fronte ad un dato importante: 14 Regioni su 15 utilizzano il metodo plurality, altrimenti noto con la formula first-past-the-post system.

L'unica eccezione è la Toscana, che utilizza invece una formula di tipo majority, nella fattispecie la majority-run off ovvero un doppio turno chiuso. Se normalmente con questa formula al secondo turno accedono i primi due competitori che hanno riportato la maggiore cifra elettorale, poiché nessun candidato ha ottenuto la maggioranza assoluta al primo turno, il caso toscano è peculiare. Difatti presenta un correttivo basato sull'attenuazione del livello di consenso percentuale da raggiungere al primo turno per essere dichiarati vincitori e accedere al premio di maggioranza più sopra esplicato: non più il $50 \%+1$ dei voti, ma il $40 \%+1$, una soglia che dovrebbe quindi limitare i casi di istituzione di un secondo turno di votazione (come è in effetti accaduto nel 2015, le prime elezioni con questo sistema elettorale, quando, appunto, non si è dovuto ricorrere al secondo turno, sebbene il Presidente Rossi avesse vinto con meno del 50\% dei voti).

Il regime delle soglie di sbarramento. - La tabella 6 sotto riportata ci mostra una panoramica alquanto diversificata per quanto concerne le clausole di accesso alla ripartizione dei seggi. Ci sembra anzitutto opportuno ricordare che la fissazione del numero degli stessi spetta agli statuti regionali, mentre l'art. 14, comma 1, lettera a) del decreto-legge n. 138 del 2011, facente parte del Titolo IV dedicato alla «Riduzione dei costi degli apparati istituzionali», ha individuato per le Regioni un tetto massimo di consiglieri regionali, in rapporto alla popolazione di ciascuna (in ogni caso da un minimo di venti a un massimo di ottanta), «mira[ndo] a garantire proprio il principio in base al quale tutti i cittadini hanno il diritto di essere egualmente rappresentati» ${ }^{17}$ senza al contempo mettere in discussione la lettera costituzionale, in quanto è lasciata alle Regioni l'esatta definizione della composizione dei Consigli regionali.

È possibile individuare infatti un primo gruppo di Regioni che nella sostanza hanno mantenuto - per questa fattispecie - l'impianto della 1. 43/1995, ossia Basilicata, Emilia-Romagna, Lazio, Liguria, Lombardia, Molise e Piemonte. Nei loro sistemi elettorali non vi è soglia legale per le coalizioni, è prevista una clausola di accesso alla ripartizione dei seggi pari al 3\% per le liste non coalizzate e non vi è soglia legale per le liste collegate al listino regionale - o ad un candidato Presidente - che abbia ottenuto almeno il $5 \%$ dei voti validi ${ }^{18}$.

La necessità di ridurre la frammentazione partitica in seno al Consiglio ha determinato, nei sistemi elettorali delle altre Regioni, la costituzione di un regime di soglie per le liste non coalizzate improntato ad una maggiore selettività [Chiaramonte 2007]:

17 Corte Cost. 17 luglio 2012 n. 198.

18 Una disposizione, quest'ultima, su cui ebbe a pronunciarsi la Corte costituzionale, che dichiarò non fondata la questione di legittimità costituzionale sollevata dal TAR Lombardia. Quest'ultimo osservava, portando ad esempio il caso delle elezioni regionali lombarde del 2013, «che l'elemento determinante ai fini dell'applicazione della soglia di sbarramento sarebbe rappresentato dal semplice collegamento della lista ad un candidato alla carica di Presidente che abbia ottenuto almeno il 5 per cento», cui la Corte obiettava ricordando che il «collegamento tra l'operatività della soglia e il risultato elettorale del candidato Presidente [...] appare coerente con la forma di governo regionale prevista dalla Costituzione per il caso del Presidente eletto direttamente, la quale valorizza il vincolo che lega il Consiglio regionale al Presidente eletto in forza del principio del simul stabunt, simul cadent», si veda Corte cost. 7 luglio 2015, n. 193. 
dal 3\% della «Tatarella» al 4\% di Abruzzo e Calabria, al 5\% della Toscana e addirittura all' $8 \%$ della Puglia; fanno eccezione - oltre a Marche e Veneto, ove non è prevista soglia esplicita alcuna per le liste non coalizzate - l'Umbria, ove vige una clausola del 2,5\% per tutte le liste, siano esse coalizzate o meno, cosa che necessariamente induce a cercare di mobilitare consenso (proprio come in Calabria, ove però è richiesto il $4 \%$, e non più il $15 \%$ dopo l'ultima modifica legislativa del 2014, intervenuta nelle more del giudizio di legittimità costituzionale richiesto dal Governo proprio in riferimento alla elevata soglia di sbarramento sopra richiamata, che avrebbe potuto «determinare una rilevante distorsione del risultato elettorale $\left.{ }^{19}\right)$, e la Campania, dove resta invariata (3\%).

Nelle Marche e in Veneto è interessante rilevare che a una lista coalizzata sia sufficiente, per entrare in Consiglio, che la sua coalizione ottenga almeno il 5\% dei voti validi, o anche una quota di consensi inferiori, purché al suo interno vi sia anche una sola lista che abbia ottenuto almeno il $3 \%$ e che di conseguenza «trascina» in Consiglio l'intera coalizione.

Il caso della Puglia è quello che presenta la maggiore selettività - si è detto - per le liste non coalizzate (8\%); di conseguenza, è maggiore la spinta a legarsi a coalizioni forti e/o ampie (che conseguano almeno l' $8 \%$ ), pur prevedendosi - anche in coalizione una clausola del $4 \%$ che di certo rappresenta un argine alla frammentazione.

Il regime delle soglie della Toscana rappresenta un caso alquanto particolare. Anzitutto, la clausola di accesso per le coalizioni è la più elevata nel panorama regionale considerato: il $10 \%$, purché almeno una lista che ne faccia parte ottenga una percentuale minima di voti validi pari al 3\%. Strettamente legata a questa è la clausola per le liste coalizzate: il 3\% se all'interno di una coalizione che superi il suddetto scoglio del $10 \%$, il $5 \%$ se la forze elettorale della coalizione risulterà essere inferiore; il 5\% rappresenta infine anche la soglia minima per le liste che corrono da sole. Da tutto ciò si può desumere che una lista forte possa - in linea di massima - optare indifferentemente per la decisione di coalizzarsi o meno: se è in grado di ottenere più del $5 \%$ dei voti, sarà infatti ammessa alla ripartizione dei seggi sia in coalizione forte, sia in coalizione debole, sia al di fuori di coalizioni. Le convenienze cambiano per una lista più debole, cui risulterà opportuno in ogni caso coalizzarsi (con evidenti implicazioni di bipolarismo): escludendo per ovvie ragioni l'eventualità di correre da sola e quindi la necessità di ottenere ameno il 5\% per poter entrare in Consiglio, essa si preoccuperà di legarsi a più liste ovvero ad (almeno) una lista forte, comunque in grado di assicurare a tutta la coalizione un totale minimo del $10 \%$, così da rendere necessario a quella lista il conseguimento del «solo» $3 \%$ dei voti, che invece salirebbe al $5 \%$ in una coalizione dalla forza elettorale inferiore al $10 \%$. 
ТАВ. 6 - Il regime delle soglie di sbarramento nelle regioni italiane.

\begin{tabular}{|c|c|c|c|}
\hline Regioni & Lista non coalizzata & Lista coalizzata & Coalizione \\
\hline Abruzzo & $4 \%$ & $2 \%$, purchè coalizione $>4 \%$ & - \\
\hline Basilicata & $3 \%$ & Presidente $>5 \%$ & - \\
\hline Calabria & $4 \%$ & $4 \%$ & - \\
\hline Campania & $3 \%$ & Presidente $>10 \%$ & - \\
\hline Emilia-Romagna & $3 \%$ & Presidente $>5 \%$ & - \\
\hline Lazio & $3 \%$ & Presidente $>5 \%$ & - \\
\hline Liguria & $3 \%$ & Presidente $>5 \%$ & - \\
\hline Lombardia & $3 \%$ & Presidente $>5 \%$ & - \\
\hline Marche & - & $3 \%$; coalizione $>5 \%$ & $5 \%$ o almeno una lista $>3 \%$ \\
\hline Molise & $3 \%$ & Presidente $>5 \%$ & - \\
\hline Piemonte & $3 \%$ & Presidente $>5 \%$ & - \\
\hline Puglia & $8 \%$ & $4 \%$ & $8 \%$ \\
\hline Toscana & $5 \%$ & $\begin{array}{l}3 \% \text { se coalizione }>10 \% \\
5 \% \text { se coalizione }<10 \%\end{array}$ & $10 \%$, purchè almeno una lista $>3 \%$ \\
\hline Umbria & $2,5 \%$ & $2,5 \%$ & - \\
\hline Veneto & - & $3 \%$; coalizione $>5 \%$ & $5 \%$ o almeno una lista $>3 \%$ \\
\hline
\end{tabular}

\section{Le modalità di espressione del voto}

In questo paragrafo esamineremo la dimensione del sistema elettorale inerente le modalità di espressione del voto. Sarà inevitabile partire dalle peculiarità regionali in materia di voto di preferenza, focalizzando la nostra attenzione sia sulle sue caratteristiche sia su quanto questa facoltà sia «estesa» per l'elettore. Vedremo i casi in cui è previsto il listino 
bloccato e quelli in cui è possibile esprimere un voto disgiunto, per poi analizzare quei meccanismi - ove previsti dalle leggi elettorali regionali - di «trasferimento» sia del voto di lista a favore anche del candidato Presidente collegato (o del listino regionale, nel caso della 1. 43/1995 ove vigente), sia del voto di preferenza a favore anche della lista e del candidato Presidente collegati, sia infine del voto per il candidato Presidente a favore della coalizione che lo sostiene. Da ultimo volgeremo lo sguardo al tema della rappresentanza di genere e ai suoi meccanismi di attuazione, facendo riferimento non soltanto - come è ovvio - alle leggi elettorali attualmente in vigore, ma anche alla normativa statale in materia (fino alle più recenti istanze legislative), nonché a pronunciamenti in merito da parte della Corte costituzionale.

Preferenze, liste bloccate, voto disgiunto. - Tutte le leggi elettorali vigenti nelle Regioni a statuto ordinario consentono all'elettore di esprimere almeno un voto di preferenza.

A questo dato di fatto aggiungiamo subito che quattro Regioni su quindici prevedono la possibilità di esprimere anche un secondo voto di preferenza: in tal caso, i candidati cui assegnare il proprio voto devono essere di genere diverso, pena l'annullamento della seconda preferenza (cfr. Tab. 7). Una di queste Regioni è la Toscana, l'unica tra l'altro a prevedere un voto di preferenza c.d. «agevolato», ossia da esprimere tracciando un segno in corrispondenza del nome e cognome del candidato prescelto, già stampato sulla scheda elettorale: uno strumento «che permette una maggiore conoscibilità dei candidati e che è stato introdotto nella normativa elettorale con l'esplicita finalità di incentivarne l'utilizzo $»^{20}$, un dato ancor più interessante se ricordiamo che la precedente normativa elettorale toscana - la 1. regionale 25/2004 - non prevedeva la possibilità di esprimere un voto di preferenza. Inoltre, se escludiamo le Regioni in cui - vigente ancora la legge Tatarella - alle elezioni si presentano listini regionali bloccati ${ }^{21}$, la Toscana è anche l'unica Regione in cui ciascun partito può facoltativamente presentare, accanto alle liste circoscrizionali, una «lista regionale» bloccata, formata da non più di tre candidati in ordine alternato di genere, i cui nomi non appaiono - in questo caso - sulla scheda e che, in caso di accesso alla distribuzione dei seggi, risultano i primi di quel partito ad essere eletti ${ }^{22}$.

Per quanto concerne il c.d. voto disgiunto, ossia la possibilità di esprimere un voto a favore di un candidato Presidente ed un voto a favore di una lista ad esso non collegata, non ne troviamo previsione nelle leggi elettorali di Abruzzo, Calabria, Marche ed Umbria, che di conseguenza «limitano la libertà di espressione del voto» ${ }^{23}$.

20 G. Bracci, «Il voto di preferenza in Toscana alle elezioni regionali del 2015», in A. Paparo e M. Cataldi (a cura di), Dopo la luna di miele: Le elezioni comunali e regionali fra autunno 2014 e primavera 2015, Roma, CISE, 2015.

21 In queste Regioni l'unica preferenza può essere espressa per un candidato inserito in una lista provinciale collegata al listino regionale stesso.

22 Art. 22, comma secondo, 1. r. 26 settembre 2014 n. 51.

23 R. D’Alimonte, «Il ‘federalismo' dei sistemi elettorali», Il Sole 24 Ore, 31 maggio 2015. 
ТАВ. 7 - Le modalità di espressione del voto nelle Regioni a statuto ordinario.

\begin{tabular}{|c|c|c|c|c|c|}
\hline Regioni & $\begin{array}{l}\text { Voto di } \\
\text { preferenza }\end{array}$ & $\begin{array}{l}N^{\circ} \text { preferenze } \\
\text { possibili }\end{array}$ & $\begin{array}{l}\text { Clausole di espressione } \\
\text { delle preferenze }\end{array}$ & Voto disgiunto & Listino bloccato \\
\hline Abruzzo & Sì & 1 & - & No & No \\
\hline Basilicata & Sì & 1 & - & Sì & Sì (regionale) \\
\hline Calabria & Sì & 1 & - & No & No \\
\hline Campania & Sì & 2 & genere alternato & Sì & No \\
\hline Emilia-Romagna & Sì & 2 & genere alternato & Sì & No \\
\hline Lazio & Sì & 1 & - & Sì & Sì (regionale) \\
\hline Liguria & Sì & 1 & - & Sì & Sì (regionale) \\
\hline Lombardia & Sì & 1 & - & Sì & No \\
\hline Marche & Sì & 1 & - & No & No \\
\hline Molise & Sì & 1 & - & Sì & Sì (regionale) \\
\hline Piemonte & Sì & 1 & - & Sì & Sì (regionale) \\
\hline Puglia & Sì & 1 & - & Sì & No \\
\hline Toscana & Sì & 2 & $\begin{array}{l}\text { genere alternato; prefe- } \\
\text { renza agevolata }\end{array}$ & Sì & Facoltativo \\
\hline Umbria & Sì & 2 & genere alternato & No & No \\
\hline Veneto & Sì & 1 & - & Sì & No \\
\hline
\end{tabular}

Infine ci sembra opportuno «immortalare» quei casi in cui le leggi elettorali regionali prevedano che una certa manifestazione del voto «si estenda» ad altri soggetti in competizione: ove sia vigente la legge Tatarella, il voto per una lista provinciale si intende espresso anche a favore del listino regionale cui è collegata; ove siano intervenute nuove leggi elettorali, si può constatare che - ovunque - il voto alle liste si «trasferisce» anche a favore del candidato Presidente che quella lista sostiene. In tre casi, votare solo per il candidato Presidente equivale a votare anche la coalizione che lo supporta: Marche, Umbria e Veneto, ove - non a caso - la competizione è fortemente coalizionale ${ }^{24}$. Ancora l'Umbria, infine, rappresenta - con la Toscana - una casistica ulteriore: il solo voto di preferenza si considera espresso a favore sia della lista di cui fa parte il candidato consigliere prescelto, sia del candidato Presidente da essa sostenuto.

24 Cfr. supra, par. 2. 
La rappresentanza di genere: la normativa di riferimento e la giurisprudenza costituzionale .- La legge 43/1995 stabiliva che nelle liste presentate in occasione dei rinnovi dei Consigli delle Regioni a statuto ordinario «nessuno dei due sessi può essere rappresentato in misura superiore ai due terzi dei candidati $\rangle^{25}$. Tuttavia questa disposizione è stata dichiarata incostituzionale dalla Consulta con una sentenza - del medesimo anno - in cui si rilevava che «l'art. 3, primo comma, e soprattutto l'articolo 51, primo com$\mathrm{ma}^{26}$, garantiscono l'assoluta eguaglianza fra i due sessi nella possibilità di accedere alle cariche pubbliche elettive $\rangle^{27}$, ragion per cui una riserva di «quote $\rangle^{28}$ posta a favore del genere sottorappresentato «non può che risultare oggettivamente discriminatoria, diminuendo per taluni cittadini il contenuto concreto di un diritto fondamentale in favore di altri, appartenenti ad un gruppo che si ritiene svantaggiato ${ }^{29}$. Proprio a questa sentenza si richiamò il Governo quando, nel 2003, propose ricorso alla Corte costituzionale contro la legge elettorale valdostana, ed in particolare contro la norma che prevedeva l'invalidamento delle liste che - tra le altre cose - non presentassero candidati di entrambi i sessi: secondo il ricorrente, infatti, la previsione della presenza in ogni lista di candidati dei due sessi avrebbe potuto essere sostanzialmente equiparata alla quota riservata all'uno o all'altro sesso. A giudizio della Corte, tuttavia, la questione era infondata, non essendo «prevista, infatti, alcuna riserva di "disuguaglianza" allo scopo di favorire individui appartenenti a gruppi svantaggiati, o di "compensare" tali svantaggi attraverso vantaggi legislativamente attribuiti» ${ }^{30}$, quanto piuttosto «un vincolo [...] alla formazione delle libere scelte dei partiti e dei gruppi che formano e presentano le liste elettorali, precludendo loro (solo) la possibilità di presentare liste formate da candidati tutti dello stesso sesso ${ }^{31}$. La stessa Corte faceva notare l'opportunità di valutare tale vincolo negativo anche in riferimento a un quadro costituzionale in parte mutato, stanti l'approvazione della 1. cost. 2/2001 - che aveva introdotto per le Regioni a statuto speciale l'obbligo di promuovere

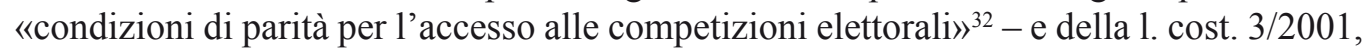
con l' «analoga, anche se non identica previsione del nuovo articolo 117, settimo comma, della Costituzione $\left.{ }^{33}\right\rangle^{34}$, e valida dunque anche per le Regioni a statuto ordinario. Tali disposizioni costituzionali avevano quindi inevitabilmente influenzato la giurisprudenza della Corte che arrivava infatti a definire «doverosa l'azione promozionale per la parità di

25 Art. 1, comma sesto, 1. 23 febbraio 1995 n. 43.

26 Nella sua formulazione antecedente la modifica del 2003, cfr. infra.

27 Corte cost. 12 settembre 1995 n. 422.

28 Cfr. infra.

29 Corte cost. 12 settembre 1995 n. 422.

$30 \quad$ Corte costituzionale 13 febbraio 2003 n. 49.

$31 \quad$ Ibidem.

32 Artt. 1-2-3-4-5, 1. cost. 31 gennaio 2001 n. 2.

33 «Le leggi regionali rimuovono ogni ostacolo che impedisce la piena parità degli uomini e delle donne nella vita sociale, culturale ed economica e promuovono la parità di accesso tra donne e uomini alle cariche elettive».

$34 \quad$ Corte costituzionale 13 febbraio 2003 n. 49. 
accesso alle consultazioni» ${ }^{35}$ - ma soprattutto aprirono le porte delle legislazioni elettorali regionali a plurime e differenti misure di attuazione delle pari opportunità elettorali ${ }^{36}$ [Milazzo 2007].

Ciò fu ancora più vero dopo la nuova modifica costituzionale operata in tale direzione dalla 1. cost. 1/2003, che al primo comma dell'art. 51 Cost. aggiungeva che «la Repubblica promuove con appositi provvedimenti le pari opportunità tra donne e uomini ${ }^{37}$; e dopo la sentenza della Corte costituzionale n. 4 del 2010, che dichiarava infondata la questione di legittimità costituzionale, promossa dal Presidente del Consiglio, dell'art. 4 , comma 3, della legge elettorale campana, in materia di rappresentanza di genere ${ }^{38}$. Secondo il ricorrente, la norma si sarebbe posta in contrasto con l'art. 3 Cost. - in quanto discriminerebbe e renderebbe disuguali candidati dello stesso sesso in caso di espressione della seconda preferenza - e con l'art. 48 Cost., in quanto «la limitazione di genere per la seconda preferenza renderebbe il voto non libero» ${ }^{39}$. Tuttavia, la Corte osservava che la norma censurata, oltre ad essere perfettamente in linea con il quadro costituzionale che legittima la finalità della pari opportunità elettorale, non altera il risultato della competizione elettorale, né la composizione del Consiglio regionale, in quanto - in via astratta - sono configurabili tutte le eventualità: squilibrio a favore di candidati uomini, squilibrio a favore di candidate donne, equilibrio di genere; da cui si deduce che «la nuova regola rende maggiormente possibile il riequilibrio, ma non lo impone. Si tratta quindi di una misura promozionale, ma non coattiva ${ }^{40}$. Con riferimento invece alla presunta violazione dell'art. 48 Cost., la Corte faceva notare che la libertà di voto non può dirsi limitata, semplicemente perché l'elettore può decidere di non avvalersi dell'utilizzo della seconda preferenza, i cui eventuali risultati non sarebbero dunque «effetto della legge, ma delle libere scelte degli elettori» ${ }^{41}$.

Oltre ai singoli e diversificati interventi che si sono di conseguenza susseguiti in ambito regionale, ci sembra opportuno rilevare più di un intervento anche sulla legislazione statale, e in particolare sulla 1. 165/2004, con riferimento alla norma che all'art. 4, in attuazione dell'art. 122, comma primo, Cost. ${ }^{42}$, individua i principi fondamentali in materia di sistema di elezione del Presidente e del Consiglio regionale, la cui disciplina di dettaglio è affidata alle singole leggi regionali ${ }^{43}$. La 1. 215/2012, «a livello amministrativo

\footnotetext{
$35 \quad$ Ibidem.

36 Nel dettaglio: infra.

37 Art. 51, comma primo, Cost.

38 «L'elettore può esprimere, nelle apposite righe della scheda, uno o due voti di preferenza, scrivendo il cognome ovvero il nome ed il cognome dei due candidati compresi nella lista stessa. Nel caso di espressione di due preferenze, una deve riguardare un candidato di genere maschile e l'altra un candidato di genere femminile della stessa lista, pena l'annullamento della seconda preferenza».

39 Corte costituzionale 14 gennaio 2010 n. 4.

$40 \quad$ Ibidem.

$41 \quad$ Ibidem.

$42 \quad$ Come modificato dalla 1. cost. 22 novembre 1999 n. 1.

43 Cfr. supra, par. 1.
} 
[...] il primo intervento a garanzia della costruzione di paritetiche condizioni tra $\mathrm{i}$ due generi in entrata alla competizione» [Agostinelli 2013, p. 34], aggiunge infatti al primo comma dell'articolo sopra richiamato un'ulteriore finalità che le leggi elettorali regionali devono perseguire: promuovere la «parità tra uomini e donne nell'accesso alle cariche elettive attraverso la predisposizione di misure che permettano di incentivare l'accesso del genere sottorappresentato alle cariche elettive ${ }^{44}$. Tali misure sono individuate da una norma di recente approvazione (1. 20/2016): essa modifica la succitata lettera c-bis), disponendo che: 1) qualora la legge elettorale preveda l'espressione di preferenze, in ciascuna lista i candidati siano presenti in modo tale che quelli dello stesso sesso non eccedano il 60 per cento del totale e sia consentita l'espressione di almeno due preferenze, di cui una riservata a un candidato di sesso diverso, pena l'annullamento delle preferenze successive alla prima; 2) qualora siano previste liste senza espressione di preferenze, la legge elettorale disponga l'alternanza tra candidati di sesso diverso, in modo tale che i candidati di un sesso non eccedano il 60 per cento del totale; 3) qualora siano previsti collegi uninominali, la legge elettorale disponga l'equilibrio tra candidature presentate col medesimo simbolo in modo tale che i candidati di un sesso non eccedano il 60 per cento del totale. In sostanza si vuole far sì che ciascuna legge elettorale regionale, ferma restando la propria autonoma competenza a disciplinare la materia nel dettaglio, tuttavia si attenga alle disposizioni di principio puntualmente individuate - per ciascuna casistica - dal legislatore statale. E proprio delle disposizioni di dettaglio delle singole Regioni tratteremo ora.

Le misure regionali di attuazione delle pari opportunità elettorali. - In tutte le Regioni a statuto ordinario, le misure introdotte per favorire - almeno nelle intenzioni - la rappresentanza di genere consistono in norme ad hoc inserite nelle singole leggi elettorali.

La più adottata (da sei Regioni) è quella delle «quote», ossia la fissazione nelle liste di soglie-limite di rappresentatività per ciascun genere: sono le c.d. gender quotas [Pezzini 2008], stabilite in tre casi (Abruzzo, Puglia, Umbria) al 60\%, negli altri tre (Campania, Lazio, Marche) ai due terzi (cfr. Tab. 8). Secondo Milazzo [2007], la scelta regionale si è orientata prevalentemente in questa direzione perché le quote sarebbero «meglio adattabili ai sistemi elettorali di tipo prevalentemente proporzionale» [Milazzo 2007, p. 108]. A tal proposito, Norris [2000] li considera in generale i più woman friendly, ove caratterizzati da ampie circoscrizioni ed assenza di voto di preferenza: le prime incentivano i partiti a formare liste bilanciate nelle loro componenti maschile-femminile, la seconda «sottrae i candidati donna $[. .$.$] ad una competizione personalistica per la quale$ sono storicamente meno attrezzati» [Chiaramonte 2007, p. 145].

44 Art. 4, comma primo, lettera c-bis), 1.2 luglio 2004 n. 165, come modificata dall'art. 3, comma primo, 1.23 novembre 2012 n. 212. 


Regioni Rappresentanza di genere Meccanismo di rappresentanza di genere

Abruzzo

Sì

Basilicata

Calabria

Campania

Emilia-Romagna

Lazio

Liguria

Lombardia

Marche

Molise

Piemonte

Puglia

Toscana

Umbria

Veneto
No

Sì

Sì

Sì

Sì

No

Sì

Sì

No

No

Sì

Sì

Sì

Sì
Lista con soglia massima di genere: $60 \%$

Norma incostituzionale e decaduta

Liste con presenza di entrambi i generi

1. Lista con soglia massima di genere: $2 / 3$;

2. Doppia preferenza di genere

1. Presenza di genere in lista massimo $50 \%+1$;

2. Doppia preferenza di genere

1. Lista con soglia massima di genere: $2 / 3$;

2. Listino regionale con presenza paritaria dei generi

Norma incostituzionale e decaduta

Liste con alternanza di genere

Lista con soglia massima di genere: $2 / 3$

Norma incostituzionale e decaduta

Norma incostituzionale e decaduta

Liste con soglia massima di genere: $60 \%$

1. Liste con alternanza di genere;

2. Doppia preferenza di genere

1. Liste con soglia massima di genere: $60 \%$;

2. Doppia preferenza di genere

1. Presenza di genere in lista massimo $50 \%+1$;

2. Liste con alternanza di genere 
Come abbiamo visto ${ }^{45}$, nelle Regioni considerate è sempre previsto il voto di preferenza, ed anche dove sia previsto il listino bloccato (ossia quello regionale ove viga ancora la legge Tatarella, e quello facoltativo toscano) esso affianca liste circoscrizionali per $i$ cui candidati è possibile esprimere almeno una preferenza. Ebbene, nelle quattro Regioni che prevedono invece la facoltà di esprimere anche due voti di preferenza (Campania, Emilia-Romagna, Toscana, Umbria) le leggi elettorali dispongono che, qualora l'elettore decida di avvalersene, le due preferenze debbano essere indirizzate a candidati di genere diverso, pena l'annullamento della seconda: è la c.d. «doppia preferenza di genere», un istituto che agisce nell'ambito del voto no seat-relevant, in quanto attiene alla competizione interna a una lista che si è già aggiudicata il seggio [Agostinelli 2013]. Nelle Regioni che la prevedono, la doppia preferenza di genere affianca sempre un'altra misura di attuazione delle pari opportunità elettorali: in due casi (Campania, Umbria) si tratta della soglia-limite sopra richiamata; in Emilia-Romagna invece affianca la disposizione - prevista anche dalla legge elettorale veneta - per cui il quantum di uomini e donne nelle liste debba essere legato al numero totale di candidati (se esso è pari, la presenza deve essere paritaria; se dispari, un genere non può superare l'altro in misura maggiore di un'unità).

Bisogna sottolineare che in questi casi nulla si dice sulla effettiva collocazione nelle liste dei candidati del sesso sottorappresentato, che, se «collocati in fondo alla lista, (hanno) minore possibilità di essere eletti» [Milazzo 2007, p. 107]; ciò è vero anche qualora sia possibile esprimere una o più preferenze, in quanto la posizione risulta appunto «rilevante perché attribuisce maggiore o minore visibilità alla candidatura e contribuisce a suggerire una gerarchia» [Pezzini 2008, p. 121]. Ovviamente la collocazione da rilevante diventa decisiva in presenza di liste bloccate: è il caso del listino regionale laziale, in cui i generi devono essere presenti in misura paritaria, ma senza indicazioni sulla posizione dei candidati, con potenziali effetti discriminatori in caso di superamento della maggioranza assoluta e conseguente dimezzamento del listino ${ }^{46}$ [Milazzo 2007].

In Toscana, invece, vige - oltre alla doppia preferenza di genere - una norma che prescrive esplicitamente la disposizione dei candidati in ordine alternato di genere, non solo nelle liste circoscrizionali, ma anche nell'eventuale listino bloccato regionale; questa norma è presente anche nella legge elettorale veneta, ed è l'unica in materia a disciplinare il sistema elettorale lombardo.

Nel caso della Calabria, invece, non solo nulla si dice sulla collocazione dei candidati, ma neppure sul quantum di rappresentatività massima dei due sessi: infatti si prescrive soltanto che le liste contemplino la presenza di entrambi i sessi, non ponendo alcun argine ai rischi di squilibrio nella rappresentanza di genere, che diventano dunque concreti. Argine non posto de jure neppure dalle Regioni in cui tale ambito non è stato oggetto di intervento da parte di nuove disposizioni di legge, per cui vige ancora la 1. 43/1995 (Basilicata, Liguria, Molise, Piemonte), la cui norma sulle quote elettorali ${ }^{47}$ - come abbiamo

45 Cfr. supra.

46 Art.3, comma quarto, 1. 23 febbraio 1995 n. 43.

47 Art. 1, comma sesto, 1. 23 febbraio 1995 n. 43. 
visto - è decaduta in seguito a dichiarazione di illegittimità costituzionale.

\section{Conclusioni}

Ciò che emerge da questa analisi comparata è che - in seguito all'applicazione del nuovo art. 122 Cost. - certamente ci troviamo di fronte a una pluralità di sistemi elettorali in minima o in larga parte differenti tra loro, ma è pur vero che sono riconoscibili alcune comuni tendenze di fondo.

La prima consiste in quello che Di Virgilio [2007, p. 240] ha definito «connubio indissolubile fra semplificazione bipolare e frammentazione»: come emerge, in particolare, nell'ambito del regime delle soglie di sbarramento - che a partire dal 1995 è stato protagonista di una sempre maggiore diversificazione -, l'intento del legislatore, sin dagli anni Novanta, è stato sicuramente restringere a due poli contrapposti la dinamica competitiva elettorale, incentivando la costituzione di coalizioni, senza però mettere in discussione, allo stesso tempo, «la sopravvivenza della preesistente variopinta serie di élite partitiche, anche marginali» [Fusaro 2008, p. 22], come si può evincere dal numero di liste che riescono ad accedere ai Consigli delle Regioni considerate, e in particolare a quelli con un numero alquanto elevato di seggi.

Coerente con la ratio delle istanze di razionalizzazione delle forme di governo locali e nazionale per via elettorale che si sono susseguite sin dal 1993, è inoltre la tendenza a conferire una potenziale stabilità alle maggioranze assembleari a tutela della governabilità della Regione, mediante la previsione - in tutte le Regioni considerate - di premi di maggioranza innestati su formule - ovunque - proporzionali. Ebbene, il paradosso è che a questo pur condivisibile intento non sempre riesce a far seguito la sua realizzazione, poiché in alcune delle Regioni in cui ancora vige la 1. 43/1995 il premio non riesce ad essere decisivo a causa di disposizioni statutarie in tal senso ostative; è forse questo l'esempio più rilevante di quanto anche istanze più recenti della storia elettorale italiana, come il premio di maggioranza, debbano fare i conti con una legge - la Tatarella - che costituisce un punto di riferimento imprescindibile anche per quelle Regioni a statuto ordinario che, in nome del «federalismo elettorale», provvedono sì ad innovare il proprio sistema elettorale, ma mantengono alcune disposizioni della 1. 43/1995.

Ciò è probabilmente dovuto ad un tipo di «federalismo elettorale» debole, ossia figlio di una competenza legislativa comunque concorrente che, in quanto tale, deve tenere conto delle disposizioni di principio individuate dal legislatore statale e - nei fatti non riesce a non tener conto del più importante prodotto normativo del legislatore statale in materia, ossia la 1. 43/1995, che evidentemente era ritenuta aver risposto adeguatamente alle esigenze del sistema politico contingente. Risulta quantomeno curioso - se non contradditorio - constatare che tale competenza rimane concorrente anche nella lettera del nuovo testo costituzionale attualmente in discussione in Parlamento; l'art. 122 Cost. infatti rimane, su questo punto, invariato, nonostante all'art. 117 il legislatore abbia provveduto a sopprimere la potestà legislativa concorrente Stato-Regioni e a razionalizzare dunque quella esclusiva statale e quella esclusiva regionale.

Non è un caso quindi che la peculiarità più rilevante della nostra analisi sia colle- 
gata ai premi di maggioranza. Da un regime di sistemi elettorali identici in ogni Regione - sul modello della 1. 43/1995, appunto un sistema misto majority assuring grazie al meccanismo premiante - rileviamo ora che 6 Regioni su 15 sono sistemi elettorali non più a maggioranza assicurata: il caso Marche, unico sistema, fra i cinque, a premio di maggioranza, e Basilicata, Molise, Piemonte, Liguria e Lazio, sistemi invece privi di un premio come precedentemente definito. Tale inedita configurazione mal si associa con la volontà dei legislatori, a partire dagli anni novanta, di agevolare processi tesi «a fabbricare o rafforzare una maggioranza (consiliare) a favore della formazione politica che ha ottenuto più voti (o seggi), così da consentire la formazione di un governo che ne sia espressione» (Chiaramonte 2011, p. 29). Certo, non una fabbricazione a tutti i costi, così come si pone il caso emblematico della Regione Marche: in questo senso, ci chiediamo se non sia un sistema a premio di maggioranza più legittimo alla luce delle motivazioni della Corte Costituzionale che hanno decretato l'incostituzionalità del premio della 1. 270/2005. Sarà dunque opportuno rilevare con maggior frequenza i possibili cambiamenti futuri in materia elettorale regionale; potremmo difatti essere di fronte ad un nuovo percorso oppure, come piuttosto ci si aspetta, in prossimità di nuove riforme che correggano tali sistemi usciti, in parte, dalla strada maestra.

E a proposito di riforme, questa volta di tipo costituzionale, sarà interessante analizzare se e in che misura i diversi sistemi elettorali regionali incideranno sulla conformazione dell'eventuale nuovo Senato, alla luce dell'art. 57 Cost. che sancisce l'elezione di settantaquattro senatori all'interno dei Consigli regionali. 


\section{Riferimenti bibliografici}

AA.VV. [2011], Elezioni e sistemi elettorali regionali. Bilancio di un quarantennio (1970-2010), Verona, Cierre.

Agostinelli C. [2013], «La doppia preferenza di genere: i suoi effetti nelle elezioni comunali del 2013 a Siena, Pisa e Massa», in Quaderni dell'Osservatorio elettorale, 70, pp. 31-58.

Baldini G. e Pappalardo A. [2004], Sistemi elettorali e partiti nelle democrazie contemporanee, Roma-Bari, Laterza.

Barbera A. e Fusaro C. [2014], Corso di diritto costituzionale, Bologna, il Mulino.

Bolgherini S. e Grimaldi S. (a cura di) [2015], Tripolarismo e destrutturazione. Le elezioni regionali del 2015, Bologna, Istituto Cattaneo.

Caciagli M. e Bolgherini S. [2008], La politica comparata. Strategie e ricerche, Le Lettere, Firenze.

Chiaramonte A. [2005], Tra maggioritario e proporzionale: l'universo dei sistemi elettorali misti, Bologna, il Mulino.

Chiaramonte A. [2006], «I sistemi elettorali delle regioni a statuto ordinari», in Caretti P. (a cura di), Osservatorio sulle fonti 2005. I nuovi statuti regionali, Torino, Giappichelli, pp 136-154.

Chiaramonte A. [2007], «Il rendimento dei sistemi elettorali regionali: un quadro comparato», in Chiaramonte A. e Tarli Barbieri G. (a cura di), Riforme istituzionali e rappresentanza politica nelle Regioni italiane, Bologna, il Mulino, pp. 221-252.

Chiaramonte A. [2011], «Il premio di maggioranza: cosa è, come varia, dove è (stato) applicato», in Chiaramonte A. e Tarli Barbieri G. (a cura di), Il premio di maggioranza. Origini, applicazioni e implicazioni di una peculiarità italiana, Roma, Carocci, pp. 15-37.

Chiaramonte A. e D'Alimonte R. [2000], Il maggioritario regionale. Le elezioni del 16 aprile 2000, Bologna il Mulino.

Chiaramonte A. e Tarli Barbieri G. (a cura di) [2007], Riforme istituzionali e rappresentanza politica nelle Regioni italiane, Bologna, il Mulino.

Chiaramonte A. e Tarli Barbieri G. (a cura di) [2011], Il premio di maggioranza. Origini, applicazioni e implicazioni di una peculiarità italiana, Roma, Carocci.

Chiaramonte A. e Vassallo, S. [2001], «Consolidare il bipolarismo limitando la frammentazione. Sobri suggerimenti sul sistema elettorale», in Le istituzioni del federalismo, n. 1, pp. 57-84.

Cosulich M. [2008], Il sistema elettorale del consiglio regionale tra fonti statali e fonti regionali, Padova, CEDAM.

Cotta M. e Isernia P. (a cura di) [1996], Il gigante dai piedi di argilla. La crisi del regime partitocratico in Italia, Bologna, il Mulino.

Cozzi A. O. [2015], «La Corte non si esprime sui premi di maggioranza regionali», in Forumcostituzionale.it.

D’Alimonte R. [2000], «Il sistema elettorale: grandi premi e piccole soglie», in Chiaramonte A. e D'Alimonte R. (a cura di), Il maggioritario regionale. Le elezioni del 
16 aprile 2000, Bologna, il Mulino, pp. 11-34.

D'Alimonte R. e Chiaramonte A. (a cura di) [2007], Proporzionale ma non solo. Le elezioni politiche del 2006, Bologna, il Mulino.

D’Alimonte R. e Fusaro C. (a cura di) [2008], La legislazione elettorale italiana, Bologna, il Mulino.

De Luca R. [2001], Il ritorno dei "campioni delle preferenze nelle elezioni regionali, in "Polis", XV, pp. 227-245.

De Luca R. [2004], Cambiamenti istituzionali e consenso. I nuovi sistemi elettorali regionali, Catanzaro, Rubbettino.

Di Giovine A. e Sicardi S. [1995], «Sistema elettorale e forma di governo a livello regionale», in Luciani M. e Volpi M. (a cura di), Riforme elettorali, Roma-Bari, Laterza, pp. 220-252.

Di Virgilio A. [2007], «Nuovo sistema elettorale e strategie di competizione: quanto è cambiata l'offerta politica?», in D'Alimonte R. e Chiaramonte A. (a cura di), Proporzionale ma non solo. Le elezioni politiche del 2006, Bologna, il Mulino, pp. 191-241.

Fabrizio D. e Feltrin P. [2007], «L'uso del voto di preferenza: una crescita continua», in Chiaramonte A. e Tarli Barbieri G. (a cura di), Riforme istituzionali e rappresentanza politica nelle Regioni italiane, Bologna, il Mulino, pp. 175-200.

Fusaro C. [1997], «La legge elettorale e la forma di governo regionale», in Barbera A. e Califano L. (a cura di), Saggi e materiali di diritto regionale, Rimini, Maggioli, pp. 223-308.

Fusaro C. [2007], «Statuti e forme di governo», Chiaramonte A. e Tarli Barbieri G. (a cura di), Riforme istituzionali e rappresentanza politica nelle Regioni italiane, Bologna, il Mulino, pp. 13-40.

Fusaro C. [2008], «I limiti della legislazione elettorale vigente», in Fusaro C. e D'Alimonte R. (a cura di), La legislazione elettorale italiana, Bologna, il Mulino, pp. $11-43$.

Luciani M. e Volpi M. (a cura di), Riforme elettorali, Roma-Bari, Laterza, 1995.

Milazzo P. [2007], «Come cambia la legislazione elettorale di contorno», in Chiaramonte A. e Tarli Barbieri G. (a cura di), Riforme istituzionali e rappresentanza politica nelle Regioni italiane, Bologna, il Mulino, pp. 93-113.

Musella F. [2011], «Il premio di maggioranza nelle Regioni italiane», in A. Chiaramonte e G. Tarli Barbieri (a cura di), Il premio di maggioranza. Origini, applicazioni e implicazioni di una peculiarità italiana, Roma, Carocci, pp. 161-182.

Nohlen D. [2010], «La riforma dei sistemi elettorali alla luce delle esperienze internazionali», in Baccetti C., Bolgherini S., D’Amico R. e Riccamboni G. (a cura di), La politica e le radici, Padova, Liviana, pp. 277-292.

Norris P. [2000], «Women's Representation and Electoral Systems», in R. Rose (a cura di), The International Encyclopedia of Elections, Washington D.C., CQ Press, pp. 348-351.

Olivetti M. [2002], Nuovi statuti e forma di governo delle regioni. Verso le Costituzioni regionali?, Bologna, il Mulino. 
Pacini M. C. [2007], «Nuovi (e vecchi) sistemi elettorali regionali», in Chiaramonte A. e Tarli Barbieri G. (a cura di), Riforme istituzionali e rappresentanza politica nelle Regioni italiane, Bologna, il Mulino, pp. 69-92.

Pasquino G. [1993], Votare un solo candidato. Le conseguenze politiche della preferenza unica, Bologna, Il Mulino.

Pasquino G. [2006], I sistemi elettorali, Bologna, il Mulino.

Pastore F. (a cura di) [2012], I sistemi elettorali regionali tra complessità delle fonti, forma di governo e dinamiche partitiche, Torino, Giappichelli.

Pezzini B. [2008], «Il riequilibrio di genere nella legislazione elettorale», in D'Alimonte R. e Fusaro C. (a cura di), La legislazione elettorale italiana, Bologna, il Mulino, pp. 111-142.

Rauti S. [2014], I sistemi elettorali dopo la sentenza costituzionale n. 1 del 2014. Problemi e prospettive, Napoli, Edizioni scientifiche italiane.

Sartori G. [2013], Ingegneria costituzionale comparata, Bologna, il Mulino.

Signorini G. [2014], La matematica delle decisioni. Sistemi elettorali, giochi e volontà popolare, Pisa, ETS.

Tarli Barbieri G. [2007], «La materia elettorale tra Stato e Regioni», in Chiaramonte A. e Tarli Barbieri G. (a cura di), Riforme istituzionali e rappresentanza politica nelle Regioni italiane, Bologna, il Mulino, pp. 41-68. 